\title{
EL IMPACTO DE LOS MOVIMIENTOS SALVEM: EL CASO DE SALVEM PARCENT
}

\author{
Juan Miguel Rafet Soriano \\ Universidad Nacional de Educación a Distancia \\ Centro asociado de Dénia
}

\section{RESUMEN}

El autor de este trabajo de investigación propone una aportación empírica que contribuya a avanzar en el insuficiente (pero no olvidado) estudio de los impactos de los movimientos sociales a partir del estudio empírico del caso de la Plataforma Salvem Parcent en el norte de la provincia de Alicante.

Palabras clave: movimientos sociales, Salvem, políticas urbanísticas.

\section{ABSTRACT}

The author of this research proposes an empirical contribution to help advance the poor (but not forgotten) study of the impacts of social movements from the empirical study of the success of the Platform Salvem Parcent in northern the province of Alicante.

Keywords: social movements, Salvem, urban policy.

\section{UNA APORTACIÓN EMPÍRICA AL ESTUDIO DEL IMPACTO DE LOS MOVIMIENTOS SOCIALES EN EL GOBIERNO DEL TERRITORIO}

Los movimientos sociales han sido objeto de estudio de diferentes ramas del saber y el conocimiento como la ciencia política, la sociología, la historia, la filosofía y la psicología social. Especialmente, en las últimas décadas, la sociología, la ciencia política y la geografía han desarrollado avances analíticos y han propuesto nuevas teorías de estudio a partir de la realización de múltiples investigaciones empíricas sobre la protesta ciudadana y los movimientos sociales. 
En las últimas décadas la evolución analítica y teórica del estudio de los movimientos sociales en la sociología y en la ciencia política se orientado en tres grandes direcciones: 1) hacia el análisis del contexto en que los movimientos sociales emergen y desarrollan sus acciones contenciosas, 2) en indagar sobre la fórmula particular de organizarse, actuar y comunicar como actor socio-político de carácter movilizador, y 3) a analizar los impactos y consecuencias que las acciones de éstos generan al actuar en la sociedad y la política.

Producto de estos avances teóricos y, sobre todo, debido al desarrollo de investigaciones empíricas sobre movimientos sociales contemporáneos (movimiento antiglobalización, pacifista, feminista, ecologista, etc.), los investigadores sociales hemos podido mejorar los análisis de este tipo de actor socio-político y dar respuestas a los principales interrogantes clásicos de investigación del estudio de los movimientos sociales: Porqué surgen, es decir, las razones del porqué los ciudadanos protestan y se organizan para tratar de modificar una decisión política, una política pública o conseguir unos objetivos o metas sociales y políticas determinadas por el colectivo ciudadano movilizado. Cómo, dando respuesta a la construcción de discursos y formas que perciben el mundo los ciudadanos que participan en la protesta o el movimiento social. Cuándo surgen, al responder y explicar cómo el contexto social y político favorece o dificulta el desarrollo de los movimientos sociales. Para qué y el porqué de la protesta y la movilización política de los ciudadanos, es decir, analizando los impactos y las consecuencias de las acciones de los movimientos sociales en la política y la sociedad durante su acción contenciosa.

A pesar de los avances analíticos de los movimientos sociales y el profuso desarrollo de investigaciones empíricas de éstos, en la actualidad existe un descuidado, pero no olvidado, marco teórico del estudio de los efectos y los impactos de los movimientos sociales.

Sorprende que el análisis de los impactos de los movimientos sociales sea uno de los aspectos menos desarrollados de la sociología y la ciencia política. Es incluso paradójico, comprobar que los ciudadanos se organizan especialmente para la consecución de unas metas o fines, aunque no exclusivamente, y rara vez, los estudios sociales mencionen la consecución de los logros o fracasos y el porqué de éstos o el porqué no de la consecución de sus reivindicaciones (Ibarra, 2005:299).

Este descuidado desarrollo analítico y las escasas investigaciones empíricas del impacto y los efectos de las acciones de los movimientos sociales son mayoritariamente debidas a: El escaso número de investigaciones empíricas que responden al interrogante de qué impactos y cómo impactan las acciones de estos actores socio-políticos en los procesos políticos, las políticas públicas y en 
la cultura política. Es muy difícil y complejo poder inferir relaciones causales directas y claras de las acciones de los movimientos sociales y sus efectos políticos posteriores, ya que son muchas las variables que infieren en acrecentar o menguar las acciones de los movimientos sociales. Resulta difícil aplicar una metodología de análisis que explique por qué las acciones de los movimientos sociales o la interacción entre diferentes actores que participan en los procesos políticos pueden llegar a modificar una política pública inicial o son capaces de cambiar una decisión gubernamental, e incluso poder transformar la cultura política de los ciudadanos. Existen una multitud de factores participantes en el proceso político en el que participan actores movilizadores y gubernamentales, y ello provoca que sea difícil mediante un marco teórico rígido comprobar cuáles son las variables que han intervenido para que determinadas estrategias, contextos y discursos políticos hayan sido fundamentales en cambios y modificaciones sustanciales en la esfera política y la sociedad. El escaso desarrollo del análisis del impacto de los movimientos sociales viene precedido por el análisis de otras tres cuestiones previas del estudio de los movimientos sociales: cómo ha evolucionado el conflicto en el que ha desarrollado su acción contenciosa el movimiento social, qué propuestas han presentado los participantes del movimiento, y por último, de qué manera han influido estas propuestas y cómo se ha modificado el contexto social en el que desarrollan su acción el movimiento estudiado (Calle, 2007:135).

Así mismo, existe el peligro de confundir «logros», éxitos» e «impactos» de las acciones de los movimientos sociales, ya que un movimiento sin conseguir sus objetivos está realizando impactos inmediatos o a largo plazo, y en ocasiones sin saberlo. El éxito de los movimientos sociales está relacionado con la consecución de sus objetivos programáticos que algunas ocasiones o no están bien determinados o son difusos. Incluso en algunas ocasiones sin conseguir la consecución de sus objetivos han logrado la modificación de algunos aspectos de la vida socio-política sin que el movimiento verdaderamente sea conocedor y sabedor de estos impactos.

Cuando los investigadores sociales abordamos el complejo análisis del impacto de los movimientos sociales queda la duda de su trazabilidad, es decir, que se produzca un resultado concreto en el sistema político que suele ser el resultado de la confluencia de diversos factores que participan en la acción de los movimientos sociales, siendo en algunos casos decisivos, intrascendentes y en otros muy difíciles de determinar cuáles han sido fundamentales para la consecución de unos impactos u otros (Casquete, 20:2011).

En España han sido varios los investigadores sociales que han resaltado la ausencia y el descuido analítico del impacto los movimientos sociales. Según 
Pedro Ibarra esa insuficiencia obedece a una serie de dificultades metodológicas en el estudio del por qué, cómo y cuándo llegan a interferir las acciones de los movimientos sociales en las políticas públicas y el tipo de gobierno desplegado durante éstas (Ibarra, 2005:299). Mientras que Ángel Calle coincide con Ibarra y Casquete al afirmar que el estudio del impacto constituye una de las dimensiones más descuidadas en las investigaciones que se realizan sobre movimientos sociales (Calle, 2007:135; Casquete, 1998).

Pero no todo son criticas sin respuesta, existen estudios como los realizados al movimiento en Defensa de los Derechos Civiles en los estados sudistas de Norteamérica, las campañas antinucleares como Campaigne of Nuclear Diasment y el movimiento para la supresión del servicio militar en España que han puesto las bases teóricas para el posterior desarrollo de teorías explicativas de los impactos políticos de las acciones de los movimientos sociales ${ }^{1}$.

En España han sido varios investigadores sociales los que han propuesto herramientas analíticas del estudio de los efectos y los impactos de los movimientos sociales a partir de investigaciones empíricas desde mediados de la década de los noventa y durante la primera década de este siglo. Varios investigadores han analizado los impactos de los movimientos antiglobalización en España, el movimiento «anti-mili» de la década de los ochenta y noventa, el movimiento ecologista en nuestro país, o más recientemente el estudio sobre los impactos de los movimientos en defensa del territorio y el movimiento del $15 \mathrm{M}$.

Entre las principales investigaciones españolas realizadas sobre el impacto de los movimientos sociales hay que destacar la investigación realizada por Salvador Martí, Pedro Ibarra y Ricard Gomà en Creadores de democracia radical. Movimientos sociales y redes de políticas públicas (Gomà et al, 2002), El impacto de los movimientos sociales. Un estudio de la protesta ambiental en España (Jiménez, 2005) realizado por Manuel Jiménez Sánchez y la propuesta de Ángel Calle en el estudio el impacto de los movimientos globales publicado en la Revista Española de Investigaciones Sociológicas (Calle, 2007). Recientemente, Jesús Casquete ha realizado un balance provisional de los logros y los impactos del movimiento español del $15 \mathrm{M}$ en el que destaca la capacidad de condicionar la agenda política como una de las primeras consecuencias de este movimiento de recién creación y análisis por parte de los investigadores sociales (Casquete, 2011).

1 McAdam: «Entre las mejoras está la valoración sistemática de James Button acerca del impacto del movimiento de derechos civiles sobre la estructura política de seis comunidades sureñas. Los datos de Button muestran claramente que el movimiento ha expandido de manera dramática las oportunidades políticas (...) (McAdam, 1998:104).» 
En los últimos años existe cierta preocupación por parte de los investigadores sociales en indagar más en las consecuencias y los impactos de los movimientos sociales. Es cierto que indagar y analizar los impactos de los movimientos sociales es una tarea ardua y complicada, aunque en los últimos años varios investigadores han empezado a desarrollarla mediante la utilización de herramientas analíticas propias de otras ramas del saber como el análisis de políticas públicas, concretamente en la fase evaluativa de los impactos de las políticas públicas.

No podremos tener un análisis completo de este objeto de estudio si no desarrollamos un marco analítico global del movimiento social que estudiamos. El objeto de estudio, el movimiento social, es un actor político de carácter movilizador que evoluciona a lo largo del proceso político y el conflicto, por lo que al mismo tiempo, se modifican los contextos socio-políticos en los que actúa el movimiento, los discursos y la forma de percibir el mundo e incluso las oportunidades y dificultades para desarrollar su acción contenciosa y conseguir sus objetivos y metas por las que se han organizado y actuado.

La aparición de nuevos movimientos, como es el caso de los movimientos globales o antiglobalización, o recientemente la movilización ciudadana en España con el movimiento del $15 \mathrm{M}$, recomienda la necesidad de realizar nuevas investigaciones empíricas que aporten un grano de arena a ese descuidado, pero no olvidado, análisis del impacto de los movimientos sociales.

La primera década del siglo XXI en España ha estado caracterizada por diversas protestas ciudadanas, siendo significativas las movilizaciones contra la guerra de Irak en 2003, las protestas contra la reforma laboral en 2002 y 2010, las protestas estudiantiles contra la Ley Orgánica de Universidades en 2001 o contra el plan Bolonia en 2009, las movilizaciones contra los efectos de la globalización y en algunos casos protestas contra la instalación de infraestructuras nocivas como el cementerio de residuos radiactivos a finales de la primera década de este siglo. Este proceso de movilizaciones en la calle en nuestro país culmina en la primavera del 2011 con el surgimiento del movimiento del 15M.

Prácticamente todos estos movimientos han tenido su trascendencia, sobre todo mediática, a lo largo de la primera década del siglo XXI en España. A parte de los movimientos mencionados, existe un movimiento a lo largo de este período de tiempo que ha desarrollado su actividad contenciosa principalmente a nivel local, pero cuyo conflicto, continuado en el tiempo e instaurado en el territorio, puede ser objeto de estudio con el objetivo de realizar una investigación empírica de éste.

A partir de finales de los años noventa y principios de la primera década de este siglo han surgido movimientos en defensa del territorio, especialmente en Cataluña y en Valencia, los denominados movimientos Salvem, que han desa- 
rrollado durante este período acciones contenciosas para paralizar el urbanismo depredador que se desarrolló en estos territorios durante la burbuja inmobiliaria en municipios catalanes y valencianos.

Por todo ello, en este artículo de investigación se realiza una aportación empírica que contribuya a avanzar en el insuficiente, pero no olvidado, estudio del impacto de los movimientos sociales, a partir del análisis del caso excepcional del movimiento Salvem Parcent en el norte de la provincia de Alicante. Los componentes de la plataforma consiguieron, temporalmente, paralizar tres actuaciones urbanísticas en este pequeña localidad al convertirse en un actor protagonista y decisor del futuro urbanismo de este municipio, debido a que varios miembros de la plataforma se presentaron a las elecciones municipales como una agrupación de electores, llegando a ganarlas, gobernar y paralizar los planes urbanísticos propuestos inicialmente por el gobierno anterior.

\section{EN BUSCA DE UN NECESARIO MARCO TEÓRICO DEL IMPACTO DE LOS MOVIMIENTOS SOCIALES EN EL GOBIERNO DEL TERRITORIO}

Partimos de la necesidad de realizar aportaciones empíricas al estudio de los movimientos sociales para poder desarrollar un marco analítico del impacto de éstos en la sociedad y en la política. Para poder desarrollar la investigación empírica del caso seleccionado en esta investigación, el estudio del movimiento Salvem Parcent en la provincia de Alicante, será necesario elaborar una propuesta analítica integradora que estudie el surgimiento, desarrollo, y especialmente, los impactos de los movimientos sociales. Varios investigadores sociales han formulado criticas sobre la rigidez de aplicación de los enfoques clásicos de estudio de los movimientos sociales y han señalado que el «corsé» de estar trabajando sólo con los movimientos sociales encorseta el estudio de dichos movimientos (McAdam et al, 2005).

Hay que añadir la existencia de una gran complejidad analítica del estudio de estos actores socio-políticos, tales como la heterogeneidad de las relaciones entre actores participantes en una política pública o proceso político, los factores del surgimiento y los impactos en la cultura política, factores que acrecientan la necesidad de un marco de análisis mucho más dinámico alejado de las frías estructuras rígidas de los enfoques y teorías clásicas.

En la actualidad nos encontramos ante la necesidad de formular una propuesta analítica que se apoye en enfoques cercanos a los análisis de las políticas públicas para poder desarrollar un marco analítico que responda de forma integral a los principales interrogantes de los movimientos sociales.

A lo largo de estas últimas décadas han sido varios los factores que han propiciado avances en la evolución analítica y teórica de los movimientos so- 
ciales en el campo de la ciencia política y la sociología. Entre los principales avances analíticos de estos actores socio-políticos cabe destacar la proliferación de investigaciones empíricas sobre movilizaciones ciudadanas que han enriquecido el marco teórico del estudio de estos fenómenos socio-políticos en la actualidad. Siguiendo a Beatriz Santamarina, la evolución analítica de los movimientos sociales puede resumirse en cuatro etapas: Una primera etapa en la que destacan los estudios sobre los movimientos sociales que se identifican con el movimiento obrero. Una segunda etapa propiciada por las movilizaciones de 1968 caracterizada por las contribuciones analíticas representadas en las teorías norteamericanas de la movilización de recursos y la europea de los nuevos movimientos sociales. Una tercera etapa, surgida a finales de la década los ochenta, caracterizada por el desarrollo analítico en ambos continentes que coincide con el aumento de movimientos sociales con el desarrollo teórico del proceso de enmarcamiento, la estructura de oportunidades políticas y el análisis de redes. Una cuarta etapa evolutiva que coincide con el estudio de los movimientos sociales que interactúan en un contexto globalizado y el debate entre institucionalización y normalización de los movimientos sociales y las distintas teorías (Santamarina Campos, 2008, 112-131).

Son varios los investigadores sociales que han sido capaces de cuantificar mediante métodos y técnicas tradicionales cuántas personas más o menos se manifiestan o salen a la calle en una manifestación o en un ciclo continuado de protestas, conflictos y reivindicaciones; averiguar mediante investigaciones cuál es la clase predominante en cada tipo de movilizaciones e incluso investigar cuáles son las variables que inciden para que los ciudadanos salgan a la calle a protestar o se movilicen con el objetivo de incidir en la toma de decisiones de un gobierno con la posibilidad de modificar cierta política pública o decisión política que afecta a un colectivo determinado o a toda la sociedad en conjunto. Estos avances analíticos se han originado también a partir del desarrollo de los principales enfoques y escuelas teóricas de la sociología y la ciencia política, especialmente el desarrollo de la hegemonía inicial de dos escuelas teóricas clásicas: la europea y la norteamericana.

El avance analítico de los movimientos sociales y la protesta pública en las últimas décadas está ligado al desarrollo de los tres principales enfoques teóricos de estudio de los movimientos sociales y la movilización ciudadana. McAdam, Tarrow y Charles Tilly, en Dinámica de la contienda política, señalan que los tres enfoques clásicos de estudio de la movilización ciudadana son: Los enfoques estructuralistas que explican la conducta de los individuos y grupos por su relación con las colectividades y centra su marco analítico en demostrar que la participación y la acción de los colectivos es fruto de las divisiones de la sociedad 
y su estructura. Los enfoques racionalistas, que centran su marco teórico en las elecciones que realizan los individuos tomando en cuenta intereses y recursos predeterminados. Los enfoques culturales que centran su análisis en la relación causal de normas, valores y símbolos que los individuos experimentan en su entorno para realizar acciones políticas grupales para la consecución de sus fines (McAdam et al, 2005:22-23).

McAdam, Tarrow y Tilly intentan retomar en su última obra conjunta las aportaciones de la agenda clásica de la teoría de los movimientos para insertarlas en un escenario dinámico acotado en el tiempo, a lo que vienen a denominar episodios, dividiendo en secuencias importantes de los procesos (a lo que ellos denominan como contienda) que se caracterizan por la conexión y concatenación de determinados elementos, que los autores denominan mecanismos. Estos mecanismos a los que hacen referencia son una serie de variables que se «toman prestadas» de los enfoques pertenecientes a las principales teorías clásicas anteriormente mencionadas. Ejemplo de ello, son la nomenclatura que utilizan al tratar los mecanismos causales ambientales procedentes del enfoque propio de la estructura de las oportunidades políticas; los mecanismos causales cognitivos relacionados con los marcos cognitivos; y los mecanismos causales relacionales propios de las estructuras conectivas (McAdam et al, 2005).

El ya clásico «Dinámica de la contienda política», supone por un lado, la acuñación de la contienda política, en la que los procesos de cambio político sólo pueden entenderse a través de la profunda interrelación que existe entre la política convencional y la no convencional; y por otro lado, el esfuerzo por proponer unas aportaciones de estudio más dinámicas del estudio de la contienda política que centrarse única y exclusivamente en un actor determinado como son los movimientos sociales.

Ante la complejidad analítica de los movimientos sociales podemos decir que no existe unanimidad ni en la percepción, ni en los contenidos, ni en las perspectivas, ni en los significados que implica el escurridizo término movimiento social, aunque la mejor estrategia posible, a la hora de delimitarlo, sea apuntar criterios amplios para luego ir adaptándolo a la variedad de movimientos y perspectivas, pero aún en todas las divergencias, en una cosa sí parece existir cierta unanimidad: en el reconocimiento del papel de los movimientos sociales pueden tener como transformadores de la realidad social, aunque a veces este papel haya sido sobrevalorado (Santamarina, 2008:113-114). Por lo tanto existen tres problemas básicos a la hora de abordar el estudio de la acción colectiva: en primer lugar, su complejidad debida a la enorme heterogeneidad en la que podemos analizar movimientos muy dispares. En segundo lugar, el gran desacuerdo existente en la delimitación conceptual y acotación de los movimientos sociales; 
y por último, a la diversidad de enfoques analíticos de un actor socio-político complejo como es el movimiento social.

Los movimientos sociales realizan acciones con la finalidad de conseguir unos objetivos o metas por los que han realizado sus protestas y acciones contenciosas en un período de tiempo determinado. Entre los objetivos de los movimientos sociales está incidir o modificar las decisiones de los gobiernos u otros actores políticos o privados que participan en un proceso político o en una política pública. Los movimientos sociales surgidos de los conflictos urbanísticos intentan mediante sus acciones incidir en las políticas territoriales o el gobierno del territorio, bien oponiéndose a determinados planes urbanísticos o proponiendo alternativas urbanísticas. En los últimos años son varios los autores que se apoyan en el marco analítico de las políticas públicas, especialmente en la parte evaluativa, para investigar la participación de los actores políticos en el diseño y elaboración de las políticas públicas.

Mireia Grau señala que en el análisis de las políticas públicas se pretende entender y explicar y medir las consecuencias tanto esperadas como inesperadas de tales acciones. Así pues, el objeto del análisis de las políticas públicas se centra, fundamentalmente, en entender y explicar el funcionamiento, las causas y las consecuencias de los procesos gubernamentales (Grau y Mateos, 2002), mientras que nuestro propósito en esta investigación es realizar aportaciones empíricas que expliquen y analicen los impactos de los movimientos sociales que interactúan en el gobierno del territorio.

Igual que sucede en el análisis de los movimientos sociales, uno de los primeros problemas que surgen cuando se pretende explicar el funcionamiento de los procesos gubernamentales es su amplitud y complejidad. Amplitud puesto que la variedad y cantidad de contextos y variables que pueden observarse cuando se analizan los procesos gubernamentales es extensa: en ellos encontramos los partidos políticos, las instituciones de gobierno, las parlamentarias, las administrativas, los distintos niveles de gobierno, pero también los grupos de presión, los ciudadanos organizados, los expertos y técnicos, los recursos económicos, la estructura social y económica del país. Y por la complejidad, complicada red de interacciones que se dan entre las variables y factores que pueden tener una incidencia en ellos. Y tal como sucediese en el análisis de los movimientos sociales, existe también una diversidad de enfoques de las políticas públicas que enriquecen el marco teórico, pero la rigidez y separación de enfoque dificulta la propuesta de un análisis más integrador. Entre los principales enfoques de análisis de las políticas públicas debemos de destacar: Una perspectiva de análisis fundamentados en variables institucionales. Aquellas perspectivas de análisis basadas en los actores y en los grupos de actores como variables explicativas de 
las políticas públicas, se trata de una perspectiva con una raíz clara en la Ciencia Política clásica que estudia las relaciones de poder, las élites políticas y el pluralismo político, y que en la actualidad ha desarrollado en análisis de redes para analizar las actuales políticas públicas. Los enfoques estructurales. Las perspectivas basadas en la acción racional. Aquellas perspectivas de análisis basadas en el papel del conocimiento de las ideas y de las coaliciones de promotores de determinadas ideas.

Mireia Grau resume estos enfoques encauzando de dos maneras el análisis de las políticas públicas. Por un lado, las políticas públicas en tanto que variables que queremos explicar, es decir, las políticas públicas como variables dependientes. En este sentido, la mayoría de las investigaciones han tendido a estudiar aspectos y fases de las políticas públicas, como por ejemplo ¿cómo y porqué se llega a configurar una política pública? ¿Qué papel juegan las redes de actores en la configuración de una política? ¿Qué factores explican las diferencias entre los contenidos de políticas de países gobernados por partidos del mismo signo político? Mientras que por otro lado, podemos investigar a las políticas públicas en tanto que variables que tienen consecuencias, con un impacto sobre aspectos de la realidad social y política y que, por lo tanto se consideran explicativas, es decir, independientes (Grau y Mateos, 2002:50).

Por lo tanto para desarrollar un marco analítico de los impactos de los movimientos sociales a partir del soporte del análisis de las políticas públicas tenemos que partir del primer enfoque señalado por Mireia Grau al entender la política pública como una variable dependiente que es modificada por la interacción de las acciones no sólo de los movimientos sociales sino también por la participación del resto de actores que participan en una política pública. En el caso de esta investigación estaríamos hablando de las políticas del gobierno del territorio, es decir, la gestión del suelo y el territorio en la que han interactuado en la última década.

Por otra parte, Joan Subirats señala que existen cuatro enfoques de análisis de las políticas públicas: Autores que centran su análisis en los procesos de toma de decisiones y en las estrategias de los actores. Esta corriente, más alejada al análisis de las políticas publicas, procede de enfoques de la sociología de las organizaciones públicas al señalar a Croizer y Friedberg. Otros investigadores más cercanos a la economía política centran su análisis en la intervención pública. Otros autores se interesan en las estructuras, procedimientos y formas institucionales de la administración pública. Este enfoque más cercano al derecho administrativo centra su análisis en las formas de funcionamiento de las instituciones administrativas y las políticas institucionales. Y por último, el enfoque cognitivo que se esfuerza en entender las políticas públicas como ex- 
presión de marcos cognitivos y normativos que constituyen sistemas de interpretación de la realidad en el seno de las cuales los distintos actores públicos y privados pueden situar sus acciones. Este enfoque centra su análisis en los principios generales, la argumentación y los valores que definen una visión del mundo que acaba reflejándose y/o reproduciendo la política pública (Subirats et al, 2008: 22-24).

Ante estas perspectivas clásicas de análisis Joan Subirats propone recoger en un marco analítico las partes más significativas de los principales enfoques analíticos de las políticas públicas, por lo que el marco analítico propuesto (Subirats et al, 2008, 28):

Aborda una política pública desde el ámbito específico de su «lógica de acción»; siendo el punto de partida la arena en la que los actores político administrativos y sociales interactúan en un ámbito determinado. Integra la influencia de las instituciones en el comportamiento de los actores mencionados y en los resultados sustanciales de la acción pública. Dedica una atención particular a los recursos que los diversos actores movilizan para hacer valer sus intereses (lo que permite una mejor combinación del análisis de las políticas públicas y las aportaciones de la gestión pública) y centra su análisis en las diferentes fases de una política pública: inclusión en la agenda, programación, implementación y evaluación.

Será a partir de la propuesta integradora del análisis de las políticas públicas realizado recientemente por Subirats y otros investigadores sociales, teniendo en cuenta la necesidad de proponer un marco analítico integrador que responda a los principales interrogantes clásicos de los movimientos sociales y poder analizar el impacto de éstos que presento las siguientes preguntas de investigación y sus consiguientes hipótesis: ¿Por qué han surgido movimientos sociales en los conflictos urbanísticos valencianos en la última década? ¿Cómo y qué ha condicionado el desarrollo de este tipo de movimientos sociales? ¿Qué impactos ha provocado esta movilización ciudadana? ¿Qué ha provocado este tipo de impactos?

Por lo que respecta a las hipótesis de por qué surgen este tipo de movilizaciones a lo largo de la primera década del Siglo XX se plantean las siguientes: A menor ordenación del territorio (planificación urbanística y políticas de desarrollo local) mayor será la conflictividad y el surgimiento de movimientos sociales. A mayor desafección ciudadana (corrupción, falta de información, representatividad) mayor conflicto en el territorio. La existencia de un cambio de valores y cultura del territorio y la aparición de una sociedad más crítica y reflexiva que busca mantener el status quo conseguido favorece el surgimiento de estos movimientos. 
Las hipótesis planteadas para responder a la pregunta de qué ha condicionado el desarrollo de estos movimientos a lo largo de esta década son: A mayor división de los niveles del gobierno del territorio mayores serán las oportunidades para desarrollar la acción contenciosa de los movimientos para cumplir sus objetivos. A mayores alianzas, mayores posibilidades de que sus demandas sean introducidas en la agenda política. Una opinión pública favorable favorece la introducción de sus demandas en la agenda política. La puesta en marcha de mecanismos participativos por parte de los gobiernos locales condicionan el desarrollo de los movimientos sociales. El sistema electoral local favorece la participación de este tipo de movimientos en las elecciones municipales. El papel de una administración supranacional con poder sancionador favorece la consecución de sus objetivos.

Mientras que los impactos más significativos son los siguientes: La inclusión en la agenda política del problema o la toma de decisión que los movimientos pretenden modificar, parar o instaurar. En la programación, concretamente en el programa de actuación político administrativo que es el conjunto de normas legislativas y actos reglamentarios que determinan los elementos sustantivos y procedimentales de la política pública o el caso concreto de actuación. Implementación, concretamente los planes de acción que son el conjunto de decisiones de planificación que determinan las prioridades en el tiempo y el espacio, así como los productos finales de los procesos políticos administrativos de los grupos-objetivos de la política pública. Evaluación, entendida como el conjunto de juicios de valor acerca de los cambios de conducta generados en los grupos objetivo y acerca de la mejoría en la situación de los beneficios finales de los impactos de las acciones de los movimientos sociales.

Y por último, cuáles son las variables explicativas que han provocado estos impactos: La modificación en el tiempo de las oportunidades políticas creadas a raíz del conflicto urbanístico. La estrategia establecida por parte del actor movilizado para poder conseguir sus objetivos (paralizar o modificar la política urbanística inicial, preservar y conservar el entorno y el paisaje, fomentar el desarrollo económico de un territorio, reivindicar infraestructuras básicas en un territorio).

\section{LOS ACTORES QUE PARTICIPAN EN EL GOBIERNO DEL TERRITORIO}

En este apartado se realiza un breve repaso sobre los principales agentes y actores socio-políticos que participan en el gobierno del territorio. Podemos observar la existencia diversa de propietarios del suelo, promotores, dueños de los medios de producción, poderes públicos y ciudadanos que interactúan en los procesos de políticas públicas territoriales como actores principales interviniendo de una 
forma u otra en la construcción de la ciudad moderna. La participación de todos estos actores públicos y privados se enmarca en un mercado libre regido por la ley de la oferta y la demanda y por las relaciones de competencia en las que predomina el carácter de dominación. Cabe mencionar que las diferencias de intereses y la fuerza de estos actores orientan sus estrategias de actuación y convierten la ciudad y el gestión del suelo en un espacio de disputa política constante en la que participan diversos actores con intereses, objetivos, recursos y estrategias claramente diferenciadas entre ellos (Zárate, 1996: 83-87).

En primer lugar, podemos identificar a los propietarios privados del suelo, cuyo principal objetivo es la obtención del máximo beneficio económico a través de la venta del suelo como mercancía. Los propietarios del suelo se aprovechan de su carácter escaso y necesario para la colectividad, de la revalorización que puede resultar de su localización dentro de la ciudad, de su aprovechamiento urbanístico y de su cambio de uso como bien económico. Mientras que los grandes propietarios buscan ante todo el beneficio que resulta de la plusvalía generada por la transformación del suelo rural en suelo urbano. Para eso se valen de distintas estrategias que intentan orientar la expresión de la ciudad hacia sus tierras. Este tipo de actor económico muchas veces recurre a parcelaciones legales dentro de los mecanismos previstos por la legislación general urbana y en cambio otras, acometen parcelaciones y urbanizaciones ilegales en la periferia, en una política de hechos consumados que obliga a las autoridades a recalificar usos del suelo para no perjudicar a terceros y a construir infraestructuras bajo la presión popular de las personas que instalan aquí su residencia (Zárate, 1996: 83).

En los conflictos urbanísticos valencianos hemos podido observar que han sido los pequeños propietarios del suelo ya consolidado los que han sufrido el proceso urbanizador, pues su objetivo no es transformar el suelo agrícola en urbano para la consecución de bienes gananciales por el cambio de recalificación urbanístico, al contrario su oposición ha sido frontal al desarrollo urbanístico e incluso a la creación de ciertas infraestructuras que modificaran el estatus adquirido o la modificación de su propiedad. Aunque existen también los pequeños propietarios del suelo que pretenden alcanzar la máxima plusvalía de sus parcelas mediante la obtención de calificaciones del suelo rentables, el aumento del potencial de edificación, el incremento de las alturas, la modificación de los volúmenes y el trazado de nuevas alineaciones. Los objetivos de los propietarios del suelo se realizan a través de la presión sobre el planeamiento oficial, en el proceso de elaboración de las políticas de ordenación del territorio y la planificación urbanística o al margen de las políticas territoriales en prácticas urbanísticas que muchas veces rozan la ilegalidad. Por lo que habría que añadir algo importante en el análisis del actual contexto urbanístico en España 
señalando que estos mismos propietarios se oponen generalmente a las medidas o políticas proteccionistas del patrimonio arquitectónico o urbanístico, porque hacen recaer sobre ellos los costes de la conservación e impiden las plusvalías a través de los mecanismos señalados anteriormente, por lo que no consiguen los objetivos de enriquecimiento generados por el cambio de tipología del suelo o la transformación del uso del suelo, como si que sucede en el caso de los grandes propietarios del suelo en el actual modelo urbanístico español.

Al mismo tiempo, la asiduidad con que los propietarios del suelo utilizan el recurso la retención de suelos urbanizables en la periferia y en el interior de las ciudades, a la espera de su revalorización por el proceso natural de expansión urbana. Con esta práctica utilizada por los propietarios del suelo éstos llegan a conseguir importantes plusvalías de los terrenos sin realizar ninguna inversión de capital ni de trabajo, por lo que la dinámica de crecimiento urbano de las ciudades provoca la transformación del suelo para beneficio del propietario del terreno y no para el beneficio de la colectividad en general (Zárate, 1996: 83-87).

Por lo que respecta a los propietarios privados del suelo como actores sociales y económicos que participan en los procesos políticos locales en las políticas territoriales, ordenación del territorio y planificación urbana, la generalización del sistema de vivienda en propiedad arraigado en las sociedades capitalistas actúa como elemento de moderación y estabilidad social dentro del propio sistema, ya que este hecho hipoteca la vida de los ciudadanos al pago de una vivienda y convierte a través del derecho de propiedad horizontal a todo comprador potencial especulador que se beneficia del aumento del valor del cambio del suelo (Zárate, 1996: 83-87).

Otro de los actores socio-económicos que participan en la configuración de las ciudades y en la gestión del suelo son los promotores y las inmobiliarias. En este caso los constructores y las inmobiliarias convierten el suelo en un producto terminado, listo para su uso mediante la promoción, la urbanización y la construcción, siendo su principal objetivo la obtención de los máximos valores de cambio a través de la venta de los terrenos edificados con el fin de recuperar cuanto antes el capital inicial invertido; por ello los promotores tienden a concentrar en sus mismas manos la construcción, financiación y venta de viviendas, de naves industriales y locales comerciales. Por ello, la participación de actores económicos en los procesos políticos de ordenación del territorio y la planificación urbana es muy variada: por un lado, existen casos en los que los promotores obtienen ayudas estables para acondicionar viviendas, sobre todo para atender a la demanda de alojamiento de sectores sociales no solventes económicamente en el marco de una política pública de fomento de la vivienda social; por otro lado, buscan la máxima edificabilidad en sus terrenos y por ello presionan el planea- 
miento oficial para conseguir aprovechamientos del suelo intensivo, mientras que crean un suelo urbano a través de la compra de propiedades rurales y de la utilización de mecanismos de expropiación y recalificación previstos por la legislación, es decir en la concreción de las políticas públicas territoriales.

Por lo que respecta a los promotores, cabe incidir en el objetivo de éstos de incrementar los beneficios especulativos, recurriendo para ello a una ocupación de la periferia a saltos: es decir, los promotores consiguen la propiedad desde los suelos más alejados, que se urbanizan y construyen primero, a los más próximos del centro. Una de las cosas más significativas de las estrategias de los promotores es realizar edificaciones en las zonas más cercanas cuando sus rentas se han revalorizado suficientemente por su situación de mayor proximidad y cuando los poderes han creado las infraestructuras y equipamientos necesarios para atender las necesidades de la población.

En el caso de los conflictos urbanísticos valencianos he de destacar que ante las conclusiones del Informe Fortu que ponía en evidencia el urbanismo desarrollado en la Comunidad Valenciana en los últimos años tras la inspección de eurodiputados en la Comunidad Valenciana, los empresarios de la Comunidad Valenciana, liderados por las organizaciones de constructores (FECOVAL ${ }^{2}$, FEVEC $^{3}$, FPIAUCV ${ }^{4}$ ) se rebelaron en tromba firmando que se trataba de la «culminación de una campaña de desprestigio, acoso y derribo a la imagen y a los intereses de la Comunidad Valenciana en Europa, orquestada por intereses oscuros y alentado por un ejercicio de irresponsable de oposición política que ha antepuesto intereses políticos a los intereses generales de la Comunidad Valenciana ${ }^{5}$.

Josepa Cucó menciona que los promotores valencianos «se arrogan la representación de los intereses colectivos de la sociedad valenciana, y copian los modos de actuar de los movimientos sociales, llegando a constituir una llamada Plataforma Cívica por la Comunidad Valenciana, cuyo objetivo es aglutinar a diferentes colectivos de la sociedad civil para defender en Europa la imagen de la región, y dar un toque de atención a las autoridades de la Unión (Cucó Giner, 2007: 6). La copia del tipo de organización de un movimiento social también se observó en las manifestaciones y reivindicaciones de la Asociación de Construc-

2 Federación de Empresas de la Comunidad Valenciana Contratistas de Obras de la Administración pública.

3 Federación Valenciana de Empresarios de la construcción.

4 Federación de Promotores inmobiliarios y Agentes Urbanizadores de la Comunidad Valenciana.

5 Comunicado de prensa emitido por la Federación de Promotores Inmobiliarios y Agentes Urbanizadores de la Comunidad Valenciana reproducido parcialmente en el periódico Levante-EMV de 15 de diciembre de 2005. 
tores y empresas a fines de Dénia, cuya constitución era modificar las políticas locales de protección del medio ambiente ante las medida del gobierno local de paralizar las licencias de obra de algunas zonas hasta que estas no tuvieran garantizada la conexión de alcantarillado ${ }^{6}$.

En cambio, en los últimos años los ciudadanos han ido apostando por la defensa del valor del uso del suelo frente a las autoridades y agentes socioeconómicos que anteponen su valor de cambio. Es la intención de los ciudadanos que los agentes políticos (gobierno, partidos políticos y oposición) resuelvan los problemas concretos de alojamiento, transporte, educación, salud y ocio, y al mismo tiempo maximicen las externalidades positivas de aglomeración y lleguen a minimizar los efectos negativos del crecimiento demográfico que han sufrido actualmente las ciudades en un mundo cada día más urbano y global. Pero a pesar de los principios filosóficos de igualdad legal y democrática, la consecución de las reivindicaciones y los objetivos de los ciudadanos en los procesos de ordenación del territorio y planificación urbanística varía considerablemente de unos a otros grupos sociales en función de variables tales como la disponibilidad de recursos materiales, el acceso a los instrumentos de planificación y la capacidad de presión e influencia en el aparato administrativo y de gobierno que atiende subsidiariamente las necesidades colectivas.

Los ciudadanos y los movimientos sociales intervienen en los procesos de ordenación del territorio y la planificación urbana a través de asociaciones y movimientos de defensa del territorio que se oponen a determinadas acciones o que intentan sacar el mejor partido posible de ellas. Podemos catalogar como ciudadanos que participan en los procesos de políticas territoriales a asociaciones de vecinos, usuarios contra implantaciones que se consideran peligrosas o perjudiciales, asociaciones en defensa de los valores ecológicos y en defensa del territorio que han llegado a convertirse en contrapoderes de las instituciones elegidas, participando en la organización de la vida local al intervenir de modo consultivo en el planeamiento oficial.

En la mayoría de los conflictos urbanísticos la oposición no está promovida en primera persona por organizaciones ya preexistentes como las primeras (propietarios del suelo, promotores e incluso partidos políticos), sino que está

6 La Asociación de constructores y empresas afines realizó una manifestación frente al Ayuntamiento en 2005 con el objetivo de que el gobierno local formado por el PSOE y el Bloc Nacionalista Valencià cambiara sus planes y les facilitara la construcción de viviendas unifamiliares no conectadas al alcantarillado pero que tuvieran fosas sépticas. El autor de esta tesis doctoral participó como secretario de los acuerdos a los que llegaron la Asociación de Constructores y el gobierno local en 2005. 
protagonizada por los ciudadanos movilizados (Cruz, 2006). Elena Cruz señala que «con el objetivo de ser un interlocutor audible para la administración, los ciudadanos se unen en una plataforma (o asociación o simplemente firmando un manifiesto) para intentar modificar un plan en cuestión (introduciendo sus propuestas) o abolirlo. Por lo tanto estos movimientos están destinados a disolver una vez acabado la contienda, ya sea porque han conseguido influir en el contenido del plan o porque éste finalmente se ha redactado sin escuchar sus protestas (Cruz, 2006:187). El Institut de Govern i polítiques públiques realizó un estudio sobre movimientos sociales en defensa del territorio destacando que «la voluntad de encontrarse, compartir experiencias y establecer cierta coordinación o un discurso propio da lugar a pensar que nos encontramos delante de la formación de un nuevo movimiento social o bien de una transformación de algunos sectores del actual movimiento vecinal y ecologista» (Alfama et al, 2006).

Oriol Nel.lo señala que los movimientos sociales propios de los conflictos urbanísticos están protagonizados por movimientos que tienden a dotarse una forma de organización específica que no coincide con ninguna entidad o actor persistente en el gobierno del territorio, y menciona que en la practica totalidad de los casos analizados la forma de organización dominante se ha denominado plataforma (...) Estas plataformas son formas de organización que surgen como reacción del anuncio de la intervención o proyecto que consideran nocivo. Nel. lo dice que los conflictos urbanísticos en los que actúan este tipo de plataformas se inician a través de la promulgación de un manifiesto o de una recogida de firmas de personas afectadas directamente por la intervención urbanística (Nel. lo, 2003: 30), hecho que sucedió en el caso seleccionado en esta investigación, es decir, en Parcent.

Por último, cabe mencionar el papel protagonista que en estos procesos realizan los poderes públicos. La administración publica orienta la expansión urbana y modela el interior de la ciudad a través de la legislación y la planificación, a través de la construcción de viviendas y la creación de infraestructuras, y a través de los usos del suelo que ella misma genera. Los poderes públicos deben realizar una importante labor de arbitraje entre los conflictos provocados por los restantes agentes económicos productores en un territorio determinado, aunque esta tarea varía dependiendo muchas variables. Los poderes públicos en sus diferentes niveles competenciales deben catalogarse como agentes de legitimación y control del orden establecido desde planteamientos estructuralistas procedentes del análisis marxista. Pero en cambio, la administración sostiene que la producción privada y la acumulación de capital es necesaria para la creación de infraestructuras y equipamientos, su participación debe ser necesaria en la elaboración 
y ejecución de programas de renovación urbana, tanto en los casos de los planes generales de ordenación urbana o planes urbanísticos como en los procesos de desarrollo territorial urbano en aquellas zonas con serias deficiencias estructurales (Zárate, 1996: 83-87).

Son muchos los autores que consideran a los poderes públicos como garantes de la reproducción de la fuerza de trabajo dentro del modelo capitalista al atender las necesidades colectivas de los ciudadanos y poder facilitar sus condiciones materiales de existencia mediante programas de bienestar social, construcción de escuelas, sistemas de trabajo social, fuerza de seguridad y mecanismos de participación pública que mantienen el orden y la cohesión social, a la vez que contribuyen a reproducir las relaciones sociales de producción. Incluso llegando a sostener que la ordenación del territorio y la planificación urbanística es la política matriz del resto de políticas públicas locales que se desarrollarán en un territorio determinado (Zárate, 1996: 83-87).

\section{LOS SALVEM VALENCIANOS EN EL SIGLO XXI}

A raíz del proceso urbanizador en España han aparecido movimientos sociales que interactúan principalmente en los conflictos urbanísticos especialmente en la Comunidad Valenciana y Cataluña. En los últimos años han surgido varios movimientos sociales con la autodenominación «Salvem» en la Comunidad Valenciana y en Cataluña. Han sido varias las plataformas contestarías y opositoras a los modelos de crecimiento insostenible del territorio que han surgido en la ciudad de Valenciana. Santamarina indica que las plataformas Salvem surgidas en la capital responderían a tres modelos: movimientos en defensa de un barrio histórico (Salvem Belluters i Salvem el Carmen), movimientos en defensa de un espacio o edificio (Salvem el Botánic y Tabacalera), y movimientos que aparecen en barrios que fueron anexionados a Valencia y sufren la amenaza de la transformación del territorio debido al proceso urbanizador vivido en España durante las últimas décadas (Santamarina, 2008: 125).

Los miembros del grupo de investigación del IGOP (Institut de Govern i Polítiques Públiques de la Universitat Autònoma de Barcelona) estudiaron a mitad la década pasada cuatro casos de movilizaciones ciudadanas surgidas tras conflictos urbanísticos en Cataluña muy parecidos a la multitud de movimientos Salvem Valencianos durante la última década (las movilizaciones contra el Plan Hidrológico Nacional, el Pla 22@bcn, la planificación urbanística y territorial de l'Empordà y el eje viario Vic-Olot en Barcelona). El grupo formado por Eva Alfama, Àlex Casademunt, Gerard Coll-Planas y Marc Martí catalogaron a los movimientos sociales surgidos de los conflictos por la ges- 
tión del suelo y el territorio en Cataluña como movimientos en defensa del territorio ${ }^{7}$.

A estos tres tipos de movimientos Salvem en la capital valenciana y los movimientos en defensa del territorio añadiría otros dos tipos de movimientos Salvem en el resto de la Comunidad Valenciana: aquellas plataformas que se han opuesto a la aprobación de planes generales y parciales que transformaban completamente la morfología territorial y social del territorio, por ejemplo la plataforma Salvem Parcent o Benassau en la montaña interior de la provincia de Alicante; y por otro lado, la aparición en la escena política valenciana de una asociación de pequeños propietarios que pedía la derogación de la Ley Reguladora de la Actividad Urbanística que se aplicaba en esta Comunidad Autónoma desde mitad de los años noventa.

Los conflictos surgidos de las políticas urbanísticas y la gestión del suelo en la Comunidad Valenciana han estado protagonizados por ciudadanos que se han movilizado para paralizar o modificar los planes urbanísticos en los municipios. El conflicto urbanístico en la Comunidad Valenciana ha llegado a traspasar las fronteras estatales. Un ejemplo de la internacionalización del conflicto urbanístico valenciano es la Resolución del Parlamento Europeo de Junio de 2006 sobre las alegaciones de aplicación abusiva de la Ley Reguladora de la Actividad Urbanística en la Comunidad Valenciana y sus repercusiones para los ciudadanos europeos, más conocido como el informe Fourtou sobre el urbanismo español.

La investigadora Helena Cruz indica que «durante los últimos años, la redacción y aprobación de planes de algunos planes urbanísticos ha desarrollado situaciones de conflicto entre ayuntamientos y los ciudadanos. La proliferación por toda Cataluña de estos conflictos, relacionados con el uso y la gestión del territorio, ha comportado la percepción de que la conflictividad entre los agentes que intervienen sobre el territorio está aumentando» (Cruz, 2006:184).

7 Los miembros del IGOP de la Universitat Autònoma Barcelona mencionaron respecto a los movimientos sociales surgidos de los conflictos urbanísticos: «si hacemos una lectura histórica de estos movimientos y nos fijamos en su evolución en un contexto social y espacial cambiante, llegamos a la conclusión que podemos hablar de un movimiento en defensa del territorio en Cataluña. El origen de este movimiento se situaría en algunas corrientes del movimiento ecologista, vecinal, que a finales de los 90 entran en un período de confluencia con la aparición del movimiento antiglobalización que da salida a un nuevo ciclo de movilizaciones en Cataluña. Los grupos que forman parte de este movimiento se caracterizan por la combinación de una «identidad proyecto» basada en una comunidad de raíz territorial con el objetivo de incidir en la política y la transformación global desde lo local, por lo se articulan a través de redes de acción colectiva críticas para conseguir sus objetivos de desarrollo alternativo. (Alfama et al, 2006: 62-63) 
El conflicto entre actores que participan en las políticas urbanísticas en el ámbito local ha provocado una serie de modificaciones en las relaciones políticas y el uso del poder, entre las que cabe señalar: La aparición y participación en el gobierno del territorio de movimientos sociales de carácter urbano en el ámbito local que son capaces de movilizar a la ciudadanía en acciones determinadas con el objetivo de interferir a su favor en ciertas políticas y planes urbanísticos desarrollados en la última década en diversos municipios catalanes, valencianos, murcianos y andaluces. La creación de una plataforma coordinadora de las acciones de los movimientos opositores al desarrollo urbanístico desmesurado en las últimas décadas con la idea de una unión mediante una red interconectada. Una «nova cultura del territorio ${ }^{8}$, como mencionan los miembros del IGOP en su trabajo realizado sobre las movilizaciones sociales relacionadas con las políticas del territorio; y por último, en el corto período de tiempo que trascurre especialmente en la última década (Alfama et al, 2006).

\section{ANÁLISIS DE UN ESTUDIO DE CASO: SALVEM PARCENT}

Parcent es un municipio alicantino de la Comunidad Valenciana en el norte de la provincia de Alicante, en el año 2007 contaba con 1061 habitantes. En la legislatura del año 2003 al 2007 este municipio alicantino estuvo gobernado por el Partido Popular. María del Carmen López Fernández, alcaldesa del PP que durante esa legislatura promovió la aprobación de tres planes urbanísticos, acción que provocó un conflicto socio-político protagonizado por la movilización de los vecinos contra estas tres actuaciones urbanísticas. A partir de este conflicto urbanístico se creó la plataforma ciudadana denominada Salvem Parcent, cuyo principal objetivo fue la paralización de los tres planes

8 Según las declaraciones de les Trobades de plataformas en defensa del territorio de Figueres y Tortosa hay que tener en cuenta: a) Mantener la biodiversidad, desarrollar una política forestal que garantice la función ecológica de los bosques y mejorar la gestión de los residuos, b) Preservar los espacios agrarios, c) Optar por un modelo energético equilibrado, d) ir hacia un modelo de máxima eficiencia y mínima ocupación del suelo fomentado el tren y los transportes públicos, e) impulsar la planificación supramunicipal que asegure un urbanismo compacto y un menor consumo del suelo y de los recursos, f) Mayor participación ciudadana en la toma de decisiones, fomentando la transparencia de la administración, mejorando el acceso de los ciudadanos a la información disponible, fomentando el debate público sobre cuestiones relacionadas con el territorio, impulsando mecanismos reales y efectivos de democracia participativa en temas de impacto territorial, y, g) fomentando mecanismos de control y sanción con la creación de juzgados especializados en materia ambiental y potenciando las fiscalías medioambientales para que velen por la legalidad vigente en materia ambiental y urbanística. 
urbanísticos. Posteriormente ciudadanos componentes de esta plataforma se integraron en la lista electoral de la coalición electoral de Ciudadanos de Parcent que consiguió ganar las elecciones municipales de mayo de 2007, formar gobierno municipal y paralizar los tres planes urbanísticos promovidos por la plataforma ciudadana que surgió con el propósito de paralizar esta actuación urbanística.

Realizando un poco de cronología de los hechos cabría mencionar que el Ayuntamiento de Parcent aprobó de forma provisional en sesión plenaria del 30 de enero de 2006 tres Planes de Actuación Integrada: «el Replà»,»la Solana» y «El Cantalar». Entre todas estas actuaciones urbanísticas sumaban unas 1800 casas y dos millones de metros cuadrados en los parajes medioambientalmente más importantes de este pequeño pueblo rural cercano a la costa mediterránea.

En la misma sesión plenaria de enero de 2006 se daba cuenta de una propuesta de concierto previo del plan general de ordenación urbana del municipio que pretendía multiplicar la población de Parcent por más de diez, sin tener en cuenta para nada el estudio paisajístico existente. Ese fue el inicio de la Asociación Veïns de Parcent, y a raíz de las protestas realizadas por unos 200 vecinos en la calle, se fue desarrollando una serie de acciones encaminadas a paralizar tales planes urbanísticos mediante la movilización ciudadana. En el mes de febrero de 2006 un grupo de vecinos acudió a una primera manifestación en Valencia: fue una de las manifestaciones más multitudinarias de los movimientos Salvem en la Comunidad Valenciana. La participación de los vecinos en esta movilización podría servir para realizar contactos con otras plataformas. El problema de Parcent empezaba a difundirse. En marzo, otro grupo de vecinos acudía a unas conferencias en Madrid. Posteriormente, el 11 de marzo se realizaba la primera manifestación pública en contra de los tres planes urbanísticos, con la participación de unas 2000 personas según los datos de los medios de comunicación. El 22 de marzo, ante la visita del Presidente de la Diputación de Alicante se celebró una concentración espontánea para evitar la inauguración de la nueva plaza del pueblo, como medida de acción de la Plataforma constituida con la finalidad de repercutir esta noticia en los medios de comunicación. El 11 de noviembre de 2006 aparece la Doctrina Parcent en una decisión sin precedentes, que ordena la suspensión cautelar del PAI del Replà por motivos de «sostenibilidad principalmente y al carecer el pueblo de Plan General de Ordenación Urbana».

El colectivo «Salvem Parcent» es una asociación que agrupa a vecinos del pueblo de Parcent en la provincia de Alicante, con un padrón de 1000 habitantes aproximadamente, que se opone a la construcción de los Planes de Actuación 
Figura 1: Imagen simulada de los tres planes urbanísticos de Parcent.
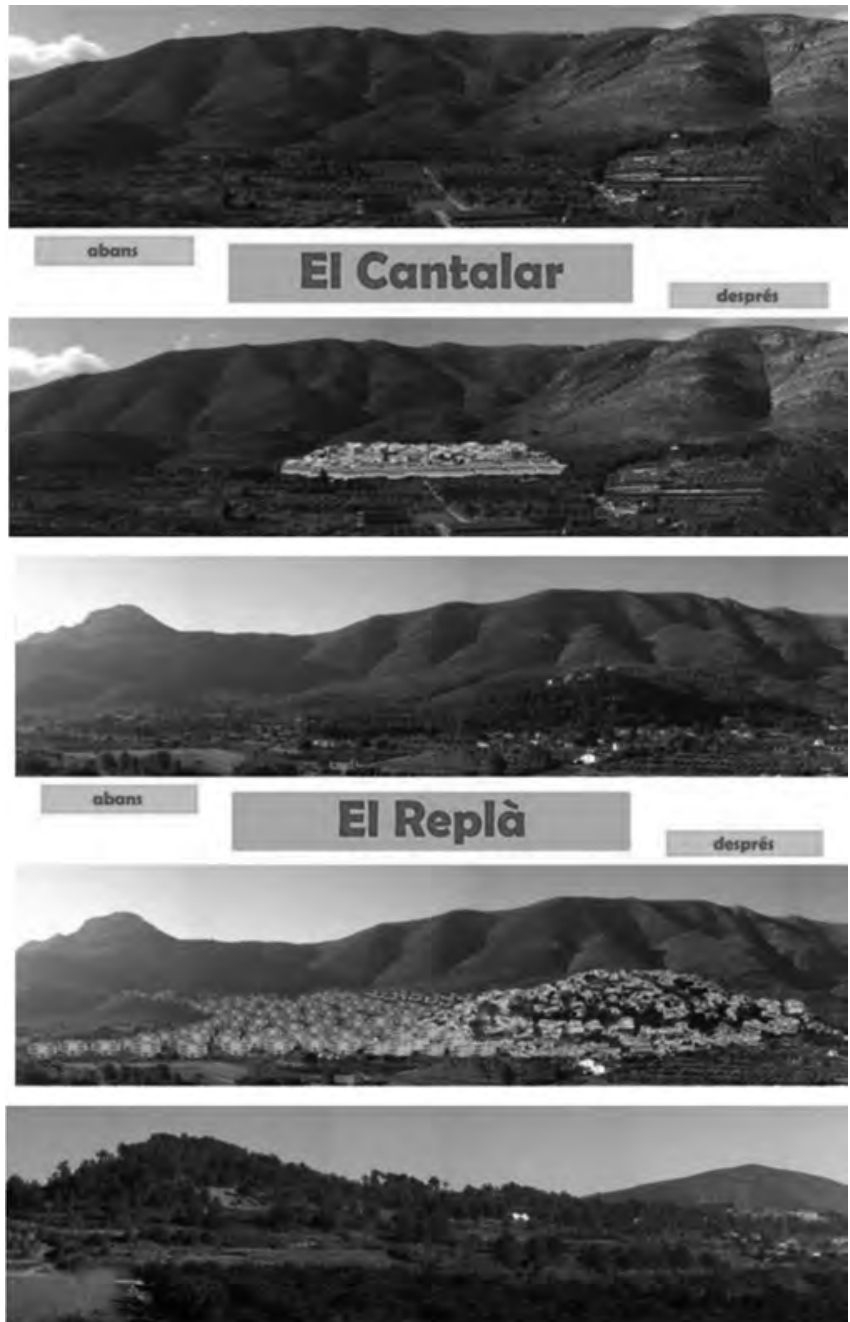

abans

\section{La Solana}

desprós

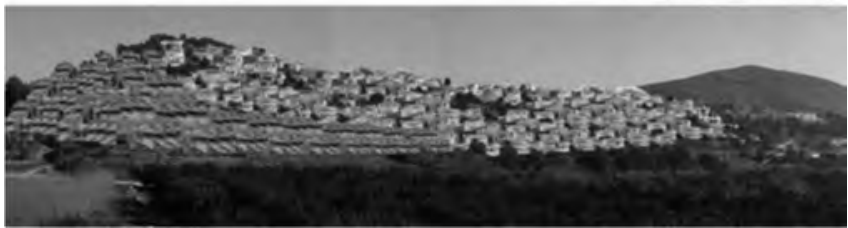

Fuente: Platafora Salvem Parcent 
Integrada aprobados por el Ayuntamiento de Parcent el 30 de Enero de 2006, planes que suponen la construcción de unas 1800 viviendas, lo que triplica el número de habitantes de esta pequeña localidad rural del norte de Alicante. Unos planes urbanísticos que pretenden desarrollar, concretamente, una de las zonas con mayor población residencial europea en toda España, la gran mayoría de ellos ingleses jubilados que han adquirido parcelas para, posteriormente, construir edificaciones residenciales como chalets, urbanizaciones o mansiones, que están afectadas por los planes urbanísticos del «Replá», «El Tosal»y «El Cantalar».

La plataforma ha realizado durante este tiempo diversas actuaciones de acercamiento a los órganos decisores, que pueden paralizar la aprobación definitiva de los tres planes urbanísticos previstos en el municipio:

- Trasladaron 1673 firmas a la Sindica de Agravios de la Comunidad Valenciana, en la que instaban a la defensora del pueblo valenciana a la retirada de los tres planes urbanísticos en Parcent. La Sindica de Agravios, sin poder legal vinculante, dió la razón a la Plataforma: "Estimamos oportuno recomendar al Ayuntamiento de Parcent y a la Conselleria de Territorio y Vivienda que se acuerde la suspensión de la tramitación de los programas de actuación integrada de El Replà, El Cantalar y la Solana y no se aprueben definitivamente hasta que no se obtenga el informe favorable de la Confederación Hidrográfica del Júcar que acredite que hay recursos hídricos suficientes".

- Recepción por parte del Grupo Parlamentario de Izquierda Unida en las Cortes Valencianas. Aunque Izquierda Unida no tiene responsabilidades de gobierno en la Comunidad Valenciana, esta entrevista con el grupo parlamentario sirvió a los miembros de la plataforma vecinal para realizar posteriores contactos con el grupo parlamentario de Los Verdes en el Parlamento Europeo, y trasladar la problemática de una población que no supera el millar de habitantes a la agenda política internacional del Parlamento Europeo.

- La presentación de un contencioso administrativo ante el Tribunal Superior de Justicia de la Comunidad Valenciana, pidiendo la suspensión cautelar del Plan de Actuación Integral de «El Replá» que origina entrar en la dinámica procesal administrativa propia de las políticas de planeamiento urbanístico español.

La «Plataforma Salvem Parcent» ha ido confeccionando y mejorando las relaciones con otros agentes sociales que pueden mejorar las condiciones de éxito de los objetivos iniciales de la asociación. Entre la mejora de las relaciones podemos señalar: 
- La constante y creciente relación con el Grupo Parlamentario Europeo de Los Verdes, que facilita el acceso por dos veces a los miembros de la Plataforma para asistir a sendas reuniones en el Parlamento Europeo de Bruselas.

- La realización de diversos contactos con la ministra de Medio Ambiente, Cristina Narbona, y el personal de su ministerio, facilitando que el problema de Parcent repercuta a niveles estatales.

- Los lazos de unión con otras plataformas o asociaciones con objetivos similares. Especialmente la relación con la Asociación «Abusos Urbanísticos No», que tiene objetivos parecidos, y está compuesta también por un número elevado de residentes europeos que han ayudado a confeccionar acciones y estrategias a «Plataforma Salvem Parcent», surgida en 2006;

- La visita realizada por un grupo de parlamentarios europeos a la costa mediterránea que origina la denuncia de la eurocámara.

Existe un escenario internacional favorable en el contexto de la Unión Europea, que favorece la consecución de los objetivos de paralización del planeamiento urbanístico del municipio de Parcent. La «Plataforma Salvem Parcent» ha sabido aprovecharse de las acciones ya realizadas en su día por la asociación «Abusos Urbanísticos No», trasladando un problema local, como son las políticas de planeamiento urbanístico, a un contexto internacional como es la agenda del Parlamento Europeo.

El factor principal de la consecución de los objetivos de la «Plataforma Salvem Parcent» ha sido la proximidad temporal de la aprobación de los tres planes urbanísticos iniciada en enero de 2006 con las elecciones municipales y autonómicas de mayo de 2007. Ya he comentado anteriormente que el Partido Popular ostentaba la Alcaldía y el gobierno local en el momento de la aprobación inicial de los tres planes, mientras que el único partido en la oposición municipal, que al mismo tiempo respaldaba la tesis de la Plataforma era la Coalición Democrática de Parcent. En el momento de la aprobación inicial de los tres planes urbanísticos, el Partido Popular tenía 4 de los 7 concejales que formaban el consistorio de Parcent. Es en el mes de abril de 2007 cuando los integrantes de la Plataforma deciden incorporase a la lista electoral de la Coalición Democrática de Parcent para conseguir la paralización de los planes desde la responsabilidad del gobierno local. Coalición Democrática de Parcent gana las elecciones municipales y Joan Carles Poquet y Jaqueline Cotteril (miembros de la directiva de la plataforma) dejan las responsabilidades en la asociación para dedicarse en pleno al Ayuntamiento, con el objetivo de paralizar los planes urbanísticos, adoptando un papel de gestor en las políticas de planeamiento a las que se habían opuesto inicialmente. 
Cuadro 1: Resultado elecciones municipales en Parcent 2007.

\begin{tabular}{|c|c|c|c|}
\hline Partido & Concejales & Votos & Porcentaje \\
\hline CDP & 5 & 378 & $52.14 \%$ \\
\hline PP & 4 & 338 & $46,14 \%$ \\
\hline
\end{tabular}

Elaboración propia

Cuadro 2: Resultado elecciones municipales en Parcent 2011.

\begin{tabular}{|c|c|c|c|}
\hline Partido & Concejales & Votos & Porcentaje \\
\hline CDP & 6 & 434 & $63,54 \%$ \\
\hline PP & 3 & 239 & $34,99 \%$ \\
\hline
\end{tabular}

Elaboración propia

Existe un dato referente a las elecciones municipales de 2007 y 2011 en la población de Parcent que quiero resaltar. Sabedores el Partido Socialista, el Bloc Nacionalista e Izquierda Unida, de la estrategia de la Plataforma de presentarse conjuntamente en la Coalición Democrática de Parcent a las elecciones municipales de mayo de 2007 con el objetivo de paralizar los planes urbanísticos del anterior gobierno del Partido Popular, deciden no presentarse a las elecciones en esta localidad para facilitar el acceso de la Plataforma al gobierno local. Por lo que este dato puede refrendar una de las hipótesis que he estado barajando a lo largo de todo el trabajo, es decir: existe una clara desafección ciudadana por la política, que se refrenda con la victoria electoral de la coalición independiente codirigida por los miembros directivos de la plataforma, y la no presentación de los partidos políticos tradicionales en el sistema de partidos políticos de Parcent.

Los miembros de la asociación de Vecinos de Parcent siempre han tenido muy claro los objetivos de su movilización, entre los que destacaré los siguientes:

- Paralizar los tres PAIS: La Solana, El replá, Cantalar.

- La anulación del pleno del 30 de enero.

- Anulación de los PAI o un referéndum al respecto.

- La creación de un Plan General imparcial que permita un crecimiento sostenible.

- La inmovilización de las cantidades que las constructoras cedan al Ayuntamiento.

- El estudio de la legalidad de los terrenos «regalados» por una promotora (2/3 de lo que regala no son suyos).

- Si se estima adecuado, iniciar los trámites para acusar de prevaricación a los responsables. 
La «Plataforma Salvem Parcent» está compuesta por medio millar de personas que respaldan las acciones encaminadas a la supresión de los tres planes urbanísticos mencionados. Se trata de una asociación legalmente constituida, que se diferencia del resto de movimientos analizados en este estudio por las siguientes características: Esta Plataforma, igual que el resto de Salvem, carece de unos recursos económicos amplios. Por lo que su acción en términos económicos es sumamente inferior al resto de actores que participan en las políticas de planeamiento urbanístico, como son las grandes promotoras ejecutoras de los Planes Urbanísticos en las ciudades. Esta discapacidad económica para desarrollar sus acciones se compensa de la siguiente forma. Defendiéndose jurídicamente desde la Plataforma, ya que algunos de sus miembros son abogados, caso que abarata los costes procesales de los contenciosos administrativos, como ya sucediese en el caso de la asociación «Abusos Urbanísticos No». Explotando el simbolismo de sus acciones principales. Una de las medidas de recaudación económica fue la venta de una camisetas con el lema «Soy de Pueblo», lo que suponía una miseria económica respecto al potencial de las empresas promotoras, pero poseía un fuerte simbolismo en sí misma. Por otro lado, trabajando en la hipótesis de que la opinión pública condiciona el impacto de los nuevos movimientos urbanos, resaltar la amplia repercusión en los medios de comunicación estatales del caso Parcent, apareciendo noticias de las acciones de la Plataforma en prensa estatal y en medios como El País o la Cadena Ser. A ello hay que añadir que las acciones de la Plataforma han tenido siempre un eco positivo en los editoriales de los principales medios de comunicación escrita de la Comunidad Valenciana. Especial referencia a este hecho es el caso del diario Levante de Alicante, que ha abierto portadas generales y publicado editoriales siempre favorables a las acciones y los logros de la «Plataforma Salvem Parcent». Resaltar que el trato con el otro diario de amplia difusión en la Comunidad Valencia, pero de editorial mas conservadora, Las Provincias, empezó a ser positivo después de la expulsión de uno de sus redactores de una sesión plenaria por parte de la alcaldesa de Parcent, defensora de los tres planes urbanísticos aprobados inicialmente.

Las acciones de la Plataforma Salvem Parcent han obtenido una repercusión política y unos impactos directos en la planificación urbanística del municipio de Parcent de tres formas distintas: En primer lugar, debido a la creación de un discurso propio de la Plataforma que se diferencia del resto de movimientos estudiados. Esto es debido a la suspensión cautelar del Plan por el Tribunal Superior de Justicia, creando la Doctrina Parcent, siendo por primera vez la justicia quien indica que no puede haber ordenación urbana a través de los Planes de Actuación Integrada sin un Plan General de Ordenación Urbana, mencionando por primera vez en una sentencia la necesidad de un desarrollo sostenible en los planes urbanísticos. Se crea el concepto de la Doctrina Parcent: por primera vez en los conflictos entre actores por las políticas de pla- 
neamiento urbanístico se refrenda mediante un auto de los tribunales la importancia de la sostenibilidad en la ordenación y desarrollo urbanístico del territorio.

En segundo lugar, la modificación del proceso político y administrativo de diseño y elaboración de los tres planes en el inicio de los trabajos de sostenibilidad, mediante la Agenda 21 Local en el municipio de Parcent. En tercer lugar, una modificación de los planes urbanísticos iniciados por el pleno de la corporación municipal en enero de 2006, debido a la victoria en las elecciones municipales de mayo de 2007 de la Coalición Democrática de Parcent, compuesta por los dos miembros más representativos de la Plataforma.

Al mismo tiempo, este tipo de acciones desarrolladas por la «Plataforma Salvem Parcent» implican la introducción de mecanismos participativos innovadores por parte del gobierno local, los cuales modifican el actual sistema organizativo del Ayuntamiento, facilitando la participación de toda la ciudadanía y agentes sociales que se consideraban no representados por la corporación municipal. Acciones que ya el anterior equipo de gobierno del PP, promotor de los tres planes urbanísticos de Parcent, inició con la introducción de los mecanismos participativos de la Agenda 21 Local, que posteriormente los miembros de la Plataforma, ahora con responsabilidades de gobierno local, fomentan en la elaboración de políticas de planeamiento urbanístico de forma participativa.

La «Plataforma Salvem Parcent» consigue con sus acciones movilizadoras impactar en las políticas de planeamiento urbanístico diseñadas por el gobierno local del Partido Popular a finales de la pasada corporación municipal. Existen una serie de factores que condicionan el éxito en la consecución de sus objetivos por parte de la plataforma. Entre estos impactos cabe destacar: el aprovechamiento al máximo del contexto internacional favorable de la Unión Europea en contra de ciertas políticas urbanística en la Comunidad Valenciana, que ya había propiciado el cumplimiento de los objetivos de la asociación «Abusos Urbanísticos No»; las buenas relaciones con el resto de actores implicados en temas de planeamiento urbanístico o movimientos sociales con fines comunes, como es «Abusos Urbanísticos No», que orientó a los componentes de la plataforma en sus acciones; la proximidad de celebración de las elecciones municipales en mayo de 2007, lo cual origina que la estrategia de la plataforma cambia radicalmente y las elites de la plataforma se conviertan en gestoras de las políticas de planeamiento, a las que hasta la fecha se oponían. También existen una serie de condicionantes que explican el impacto de la movilización ciudadana en Parcent y sus éxitos, entre los que cabe destacar: la realización de lazos de unión y configuración propia de la plataforma, tanto de ingleses como residentes originarios de la zona norte de Alicante, es decir, las buenas relaciones entre el colectivo inglés, asentado desde hace unos años en el litoral mediterráneo español, y los residentes valencianos que 
ahora tienen las propiedades de su pueblo como segunda residencia. Este tipo de movilización ha provocado una serie de impactos en el sistema organizativo del gobierno y la administración local en varias ciudades, como son la implantación de sistemas participativos y de consulta en temas de gran importancia para el municipio, como son las políticas de planeamiento urbanístico.

\section{CONCLUSIONES}

En este trabajo de investigación se ha propuesto nuevas aportaciones analíticas al estudio de los impactos los movimientos sociales a partir de la investigación empírica del movimiento Salvem Parcent en el marco de las políticas urbanísticas desarrollistas que han caracterizado al urbanismo español en la última década.

Más allá de la oposición y paralización de los planes urbanísticos por parte de la plataforma analizado cabe resaltar unas consideraciones finales del estudio de caso de los Salvem Valencianos: 1. El estudio de caso refleja la conciencia colectiva de la ciudadanía ante las agresiones al medio físico que les envuelve. Esta conciencia va ligada a la existencia de un nutrido grupo de residentes extranjeros en Parcent con una cultura del territorio más respetuosa con la protección del paisaje y alejada del urbanismo desarrollista neo-liberal que ha caracterizado el urbanismo en las últimas décadas. 2. Estamos en un caso excepcional en el que los ciudadanos movilizados en contra de los planes urbanísticos se han convertido en actores públicos principales de la política urbanística a la que se oponen, debido a la estructura de oportunidades políticas relacionadas con su victoria en las elecciones municipales de 2007 y su revalida electoral en 2011 tras paralizar los tres proyectos urbanísticos e iniciar la redacción de un nuevo plan general.

Es importante destacar en la conclusión que el caso Parcent, a pesar de ser un micro-caso de la conflictividad urbanística valenciana de las últimas décadas, refleja la movilización ciudadana ante el urbanismo desarrollista, pero sobretodo, la consecución de las metas y objetivos de los ciudadanos y sus impactos, más allá de sus objetivos iniciales de paralización de los tres planes urbanísticos.

\section{BIBLIOGRAFÍA}

Alfama, E. Casademunt, A. Coll-Planas, G. Cruz, H. (2006): Per una nova cultura del territorio? Mobilitzacions i conflictes territorials. Barcelona: Icaria.

Blanco, I. y Gomà, R. (coords.) (2002): Gobiernos locales y redes participativas. Barcelona: Ariel.

Calle, A. (2007): El estudio del impacto de los movimientos sociales. Una perspectiva global, en Revista de Investigaciones Sociológicas $\mathrm{n}^{\circ} 120$, págs. 133-153. 
Calle, A. (2005): Nuevos movimientos globales. Hacia la radicalidad democrática. Madrid: Editorial Popular.

Casquete, J. (1998): Política, cultura y movimientos sociales. Bilbao: Bakeaz.

Casquete, J. (2011): «Balance preliminar de la indignación», en Viejo Topo $n^{\circ}$ 286, 2011, págs. 19-25.

Coller, X. (2000): Estudio de Casos. Colección Cuadernos Metodológicos nº 30. Madrid: CIS.

Cruz I Gallach, H. (2006): «Conflictos territoriales y movilizaciones ciudadanas: algunas reflexiones sobre las formas de gobernanza territorial actuales», en Boletín de la Asociación de Geógrafos Españoles, núm. 48, p. 375-387.

Cruz I Gallach, H. (2010): La veu de la ciutadania en el planejament urbanistic. Una reflexió sobre la conflictivitat territorial a Catalunya, Barcelona, Tesis Doctoral inédita, Universitat Autónoma de Barcelona.

Cucó, J. (2007): «Urbanización y revuelta. Aproximación al caso de la ciudad de Valencia». En prensa.

Grau, M. Mateos, A. (2002): Análisis de Políticas Públicas en España: enfoques y casos. Valencia. Tirant lo blanc.

Godàs Pérez, X. (2007): Política del disenso. Sociología de los movimientos sociales. Barcelona: Icaria.

IbarRA, P. (2005): Manual de sociedad civil y movimientos sociales. Madrid. Editorial Sintesis.

IbarRa, P. Martí, S. y Gomè, R. (coords.) (2002): Creadores de democracia radical. Movimientos sociales de políticas públicas: Barcelona. Icaria.

Jiménez SÁnchez, M. (2005): El impacto político de los movimientos sociales. Un estudio de la protesta ambiental en España. Madrid: Centro de Investigaciones Sociológicas.

McaDAm, D. (1998): Orígenes conceptuales, problemas actuales y direcciones futures. Los movimientos sociales. Transformaciones políticas y cambio cultural. Madrid: Editorial Trotta.

Mcadam, D. Tarrow, T. y Tilly, C. (2005): Dinámica de la contienda política. Barcelona. Editorial Hacer.

Santamarina Campos, B. (2008): Movimientos sociales: una revisión teórica y nuevas aproximaciones. Boletín de antropología, Vol 22, Núm.39, pp $112-$ 131. Universidad de Antíoquia. Colombia.

Subirats, J. Knoepfel, P. Larrue, C. Varonne, F. (2008): Análisis y gestión de políticas públicas. Barcelona. Ariel Ciencia Política.

Teluis, W. (1997): Introduction to Case Study. The Qualitative Report, Volume 3. ZárATE, A. (1996): Ciudad, transporte y territorio. Madrid: Cuadernos de la UNED. 